\title{
Supervision system of english online teaching based on machine learning
}

\author{
Wen Lu' ${ }^{1}$ G. N. Vivekananda ${ }^{2}$ A. Shanthini ${ }^{3}$
}

Received: 28 April 2021 / Accepted: 29 December 2021

(c) Springer-Verlag GmbH Germany, part of Springer Nature 2022

\begin{abstract}
The automated supervision system for online teaching is volatile in current teaching observation. Hence, it requires additional comprehensive, analytical, and realistic discussion on how the automatic supervision method can be applied to high school teaching. This paper integrated remote supervision with machine learning algorithms (IRS-MLA) proposed for the online English teaching audit process. Here, IRS-MLA simulates the implementation of supervision methodologies in the teaching process according to English online teaching's real needs. Furthermore, searching the performance and stating the learning process for students from the teachers' perspectives and their students measures the teacher's teaching process. This paper presents the studies for evaluating the classic English language online supervision and explores this method's functional impact. This analysis's findings show that the model developed in this paper worked well and validated based on the case study report. This study validates the proposed IRS-MLA with the highest performance ratio of $97.8 \%$, the accuracy of $96 \%$, the efficiency of $99.3 \%$, and a success ratio of $98 \%$, compared to existing models.
\end{abstract}

Keywords Online teaching $\cdot$ Machine learning $\cdot$ Teaching process $\cdot$ Supervision

\section{Overview of english online teaching}

The modern health emergency influences the behavior of society in various ways [1]. Speed up Internet technology (IT) has become an essential education and teaching reform activity for education, IT training and online networking [2]. In recent times, online teachers have become a hot trend that has shown that all fields of education, health, industry, the internet, and the like are vulnerable [3]. Unlike a classroom experience, a series of computer-based teach-

$\bowtie \quad$ Wen Lu

wendy0011@126.com

G. N. Vivekananda

vivekanandagn@gmail.com

A. Shanthini

shanthia@srmist.edu.in

1 College of Foreign Studies, Guilin University of Electronic Technology, Guilin 541004, Guangxi, China

2 Madanapalle Institute of Technology and Science, Madanapalle, Andhra Pradesh 517325, India

3 Department of Information Technology, College of Engineering and Technology, SRM Institute of Science and Technology, Chennai, Tamil Nadu 603203, India ing procedures, including computer resources and software, involves an online learning experience [4]. This online learning model helps students progress, makes it more flexible in choosing their training, provides new opportunities for collaborative projects and concentrates on evaluation and feedback [5].

A Machines Learning (ML) system for artificial intelligence (AI) can interact and monitor student performance. More algorithms are used to identify patterns to predict results [6]. ML algorithms will determine whether a student has problems with a concept continuously. The system can change the contents of e-learning to allow the student to access further information [7]. Therefore, there are specific techniques for an online student who hasn't mastered the basic concepts required for continuation. [8]. Students spend less time online with machine learning assistance because they provide psychological support with their machine training software, leading to more online graduates [9].

In other words, however, precursor teachers should incorporate technology into their careers to reducing the gap between professors and the use of technology [10]. Therefore, the authors consider establishing an electronic monitoring system for teachers and supervisors can communicate in a more practical way (E-Supervision) [11]. E-Supervision 
refers to the use of videoconference technologies in real-time [12]. Since the e-supervisor has a different physical position than its supervisor, video conference technology enables the e-supervisor to perform several professional jobs [13] successfully. Supervisory can help the supervisor professionally improve the growth of the supervisor and further education [14].

Therefore, evaluating supervisory services' role in improving the learning process is crucial [15]. Our mission is to offer quality-free innovation and protect and support students' academic activities [16]. However, the automated English education method is not reliable in the current teaching assessment [17]. Therefore, the challenges and adaptive models of education must be identified, new and improved technologies incorporated to continue to learn in any situation. [18].

Moreover, the pyramid of education is not achieved by an unreasonably high proportion of university students [19]. The secondary vocational study is not conducted as planned, and the balance of primary school teachers receiving training is increasing marginally [20].

The main contribution of the paper is.

- Attempts to make the learning process more effective using integrated remote supervision with machine learning algorithms (IRS-MLA).

- Improves to identify training and learning gaps, utilizing analytical data and reporting using a hybrid learning management system (HLMS).

- Attempts to make the learning process more effective using integrated remote supervision with machine learning algorithms (IRS-MLA).

- Improves the educational collaboration atmosphere among supervisors and professors, which involves gaining more skills, experiences, attitudes, and teaching strategies.

Section 1 deals with the importance of the online effective teaching process and a detailed literature review in Sect. 2. The integrated remote supervision with machine learning algorithms (IRS-MLA) is proposed for effecting learning, evaluation and E-supervision is discussed in Sect. 3. The experimental results are given in Sect. 4, and finally, it concludes with Sect. 5 .

\section{Literature review}

Panel Choudriea et al. [21] proposed Machine learning strategies MLS to distinguish knowledge and misinformation about COVID-19. The paper breaks new ground by stressing differences in the way human information is processed. This ability is still empirically checked. A vulnerable demo- graphic sector provides insights into connections to online knowledge and disinformation in an early pandemic. The paper reflects a cross-section of two rather disparate ML methods and interviewed data through thematic analyses and grounded theory principles that enhance understanding of human and modern technological interaction.

Zhai et al. [22] described computerized technology machine learning (CTML) based on algorithms built from training rather than "education" data that have a great potential for revolutionizing scientific assessment. Three vertices with technique, validity and educational features were used in the evaluation of ML science. It is found that most studies focused on the reality of vertex compared with the other two vertices. Current research involves text recognition, classification and assessment, focused on scientific explanation, with a broad range of human-machine agreement measures. Most studies used a cross-validation method, not self-validation or split validation, to achieve contractual standards. ML enables teachers to use complex assessments and save time and cost without the burden of human costs. Most studies conducted ML based on the extraction of human attributes first coded by students from work instead of nonconducting or semi-conducting ML.

Han [23] invented remote supervision algorithms and deep learning (RSA-DL) to incorporate a framework structure for the online assessment method in English, further simulates and analyzes the application in monitoring the teaching process. The paper measures the conduct and status of the learning process in the teacher evaluation and student assessment and evaluates the teacher's teaching process. This paper provides experiments to test the English learning model online to examine this practical impact on English's online teaching. Research shows that the paper's model is adequate.

Villegas-Ch et al. [24] introduced machine learning and learning management systems (LMS) to suggest integrating learning management systems of technology, such as artificial intelligence and data processing, to enhance learning. This objective is outlined in a new way. Several activities are done online, and technology gives students virtual assistance to guide them in their training. Strong pedagogical models are sought.

Zawacki-Richte et al. [25] proposed artificial intelligence in higher education (AIEd) to provide a comprehensive analysis of research on AI applications in higher education. The synthesis of results shows four areas of AIEd applications to support academic, institutional and administrative services. These conclusions reflect the near lack of critical reflection on AIEd's threats and challenges, the weak connection to theoretical educational perspectives and the need to further study AIEd's higher education application through ethics and educational approaches.

Wu et al. [26] proposed supervised Machine Learning (SML), which compares the efficiency of classification and 
consistency with human coding and ML algorithms. Students had higher final classes with more messages supporting statistically two or more ML algorithms. All three ML algorithms approved students that failed significantly less than study participants. The results suggest that ML can identify and monitor extensive educational data in the intimate learning environment for students in need of support.

According to the literature survey, the proposed paper rectifies the problems using integrated remote supervision with machine learning algorithms (IRS-MLA). Furthermore, a Hybrid learning management analysis is introduced to enhance the performance and stating the teaching process for students. The intention is, therefore, to discern and improve the learning process.

\section{Integrated remote supervision with machine learning (IRS-ML)}

Online learning or virtual courses are offered via the internet compared to conventional lessons at the brick and mortar school. It has started with the spread of the Internet and the World Wide Web in the mid-1990s as the latest distance learner development. The learner's experience is asynchronous as well as synchronous. Teachers of the English language can employ difficult evaluations using ML and save time and money without incurring human costs. Instead of non-conducting or semi-conducting ML, most research used ML to extract human qualities initially coded by students from work.

Figure 1 depicts online teaching where students gain knowledge about various innovative projects in numerous fields, building constructions, astronauts, research field, chemist, medical analysis etc.,

A pupil spontaneously communicates with other students to develop awareness. The student may learn from others through his or her experiences with other students. Two students, $\mathrm{Y}$ and $\mathrm{Z}$, are randomly chosen from the student population, and the result comes from Eq. (1).The selected learners, $\mathrm{Y}$ and $\mathrm{Z}$, have their learning result $A_{\text {total }}^{1}-Y$ and $A_{\text {total }}^{1}-Z$ which are the respective updated values of $A_{\text {totalnew }}^{1}-Y$ and $A_{\text {totalnew }}^{1}-Z$ at the end of the teacher phase.

$S_{r, Y, j}^{m}=S_{r, Y}^{m}+a^{j} * 1$

where

$l=\left\{\begin{array}{l}S_{r, Y}^{1}-R_{r, Z, j}^{1} \text { if } A_{\text {total }}^{1}-Y, j<A_{\text {totalnew }}^{1}-Z, j \\ S_{r, Y}^{1}+R_{r, Z, j}^{1} \text { if } A_{\text {total }}^{1}-Y, j>A_{\text {totalnew }}^{1}-Z, j\end{array}\right.$
From Eq. (1) $a^{j}$ indicates the teaching methods used in internet technology and further.

$S_{r, Y}^{m}, S_{r, Y}^{1}$ intervenes about the learning segmentation form number of students and for the single student. Furthermore, the quality of teaching can be improved by maintaining the values of $R_{r, Z, j}^{1}$ to a standard value obtained from Eq. (1). Thus the learning result $S_{r, Y, j}^{m}$ after interactions are derived from Eq. (1). A person who intervenes to alter the control algorithm is sometimes observed if it is considered appropriate and followed as the directive; on the other hand, it independently records the results and expects further guidance. Using technology to improve and assist the educational environment is referred to as integrating technology. Classroom technology integration can help students by allowing them to accomplish computer work using standard pencils or papers. Students may effectively integrate technology when they can pick technology instruments to get information, evaluate and synthesize it swiftly, and deliver it to a realworld audience. Technology should play a role in how the classroom functions as accessible as any other teaching tool.

\subsection{Hybrid learning management system}

A Hybrid Learning System (HLMS) is a software framework used to manage, document, record, report, automate and deliver education, education and development courses. E-learning directly develops the definition of the hybrid learning system. While the first HLMS was established in the high-level education sector, most HLMS focuses on business. For apprenticeships, HLMS is the leading market segment.

HLMS is used to construct organized technical material for training. Figure 2 depicts various HLMS algorithm activities, including online education, supervision, follow-up and qualifications, training, monitoring, and teaching. Additionally, it is used for adding text, photos, videos, PDFs, tables, text, appositives, interactive testing, etc. Similarly, different people can be generated, such as teachers, students, parents, visitors and publishers. It monitors information whether a student can access, monitors progress and uses communication instruments. Teachers can take courses and modules, register or register, view student reports and put students online.

HLMS may allow teachers to create individual tests for students, both accessible and submitted online. The multimedia response, multi-choice response, drag and fall order, trial, real or fictitious/yes and no; gaps filling, agreement scale and offline tasks are the basis for a variety of questions. Various HLMSs allow participation and integration into classrooms where administrators can see if they attend, arrive late, or skip classes and meetings. Feedback can be exchanged via HLMS from both students and their peers are the best solution to improve online education performance. Therefore, the performance of an algorithm is assessed using two metrics, 


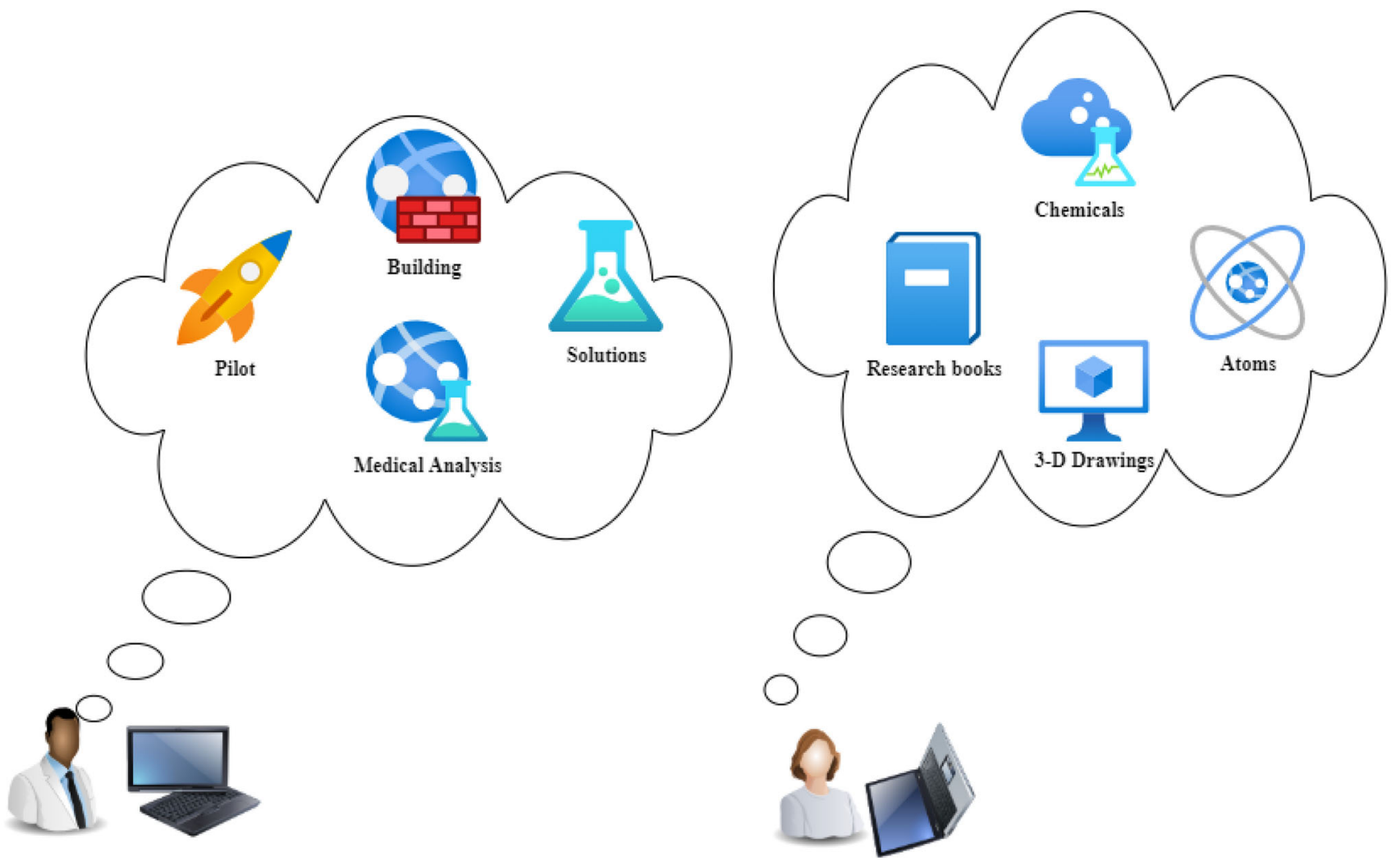

Fig. 1 Online learning

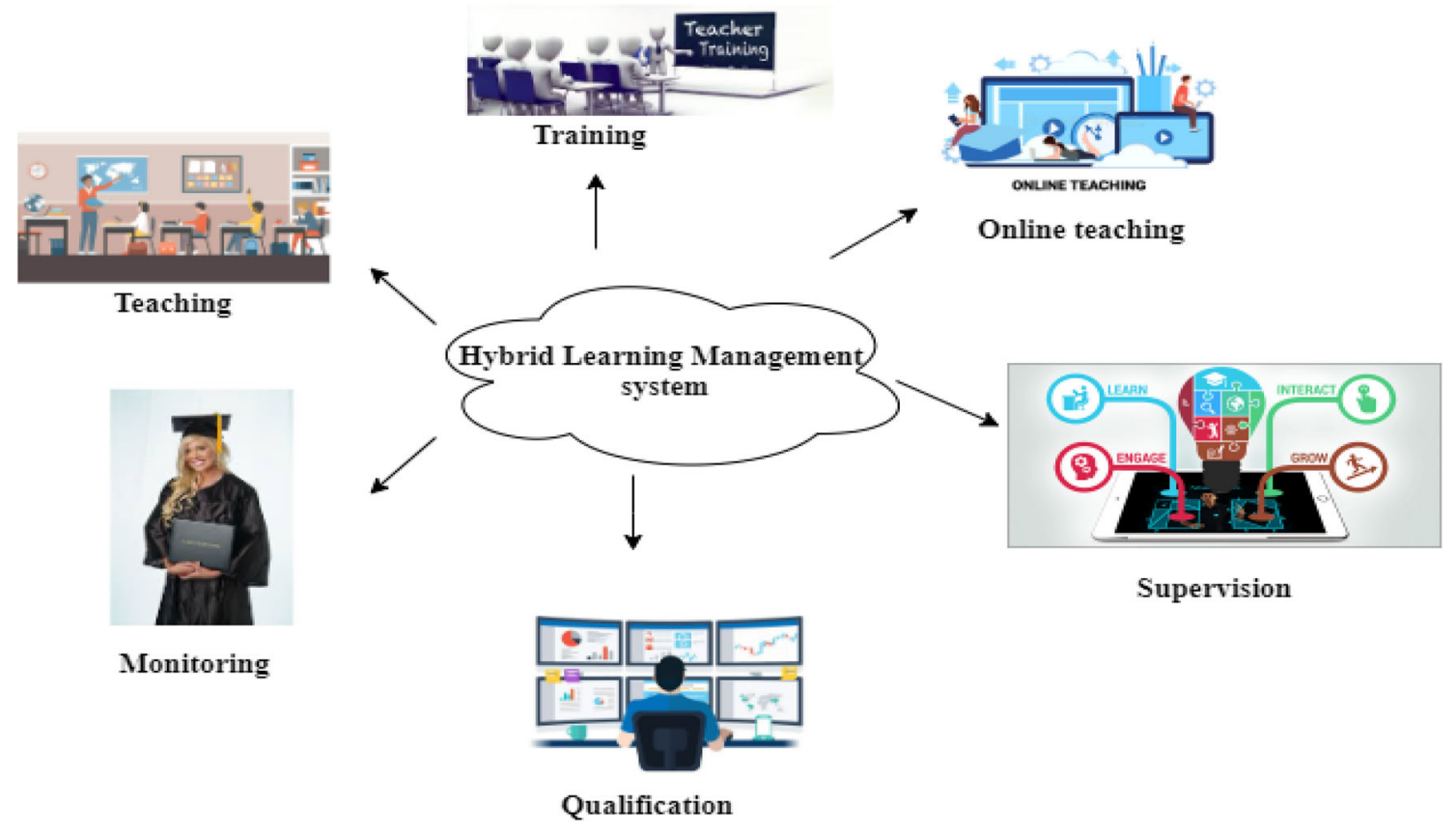

Fig. 2 Hybrid- Learning Management System

metric $E J_{R}$ is applied to measure the performance of the students learning $\Omega^{l}$ relative to a teacher's teaching $\Omega^{*}$ is explained in Eq. (2)

$$
E J_{R}\left(\Omega^{l}\right)=\frac{1}{\Omega^{*}} \sum_{y \in \Omega^{l}} \min \left\{e_{x, y} \mid x \in \Omega^{l}\right\}
$$


Fig. 3 Performance of the Students

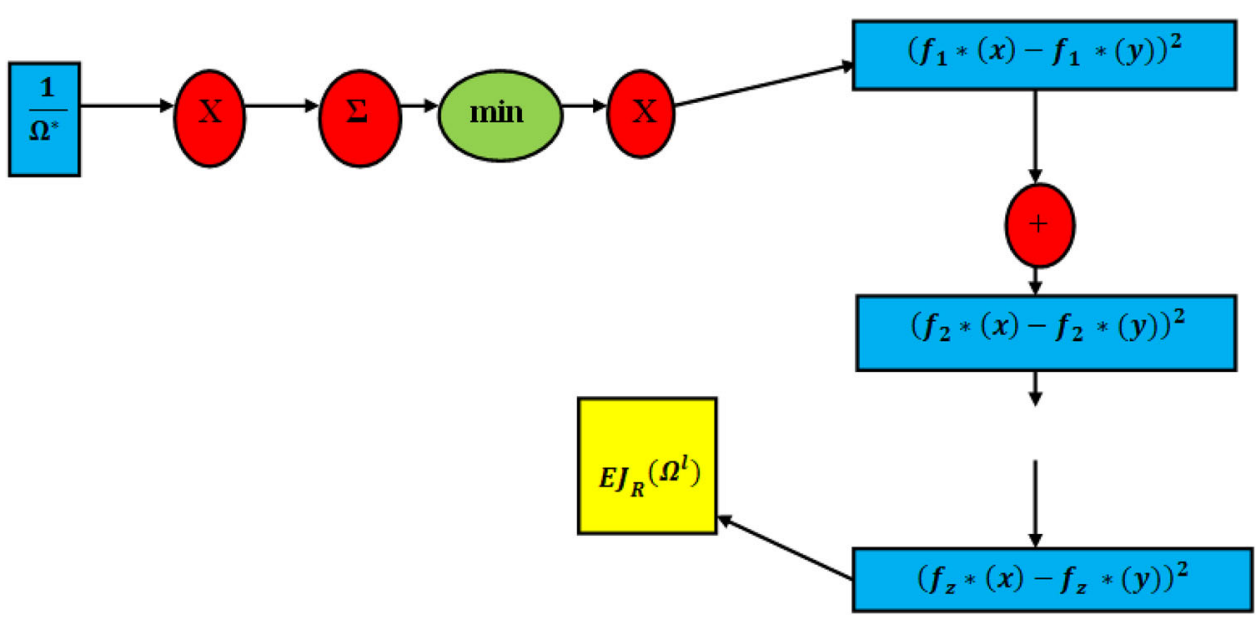

As inferred from Eq. (2) $e_{x, y}$ is the performance between a solution $x$ and a reference solution $\mathrm{y}$ in the normalized objective space has been described.

$$
e_{x y}=\sqrt{\left(f_{1} *(x)-f_{1} *(y)\right)^{2}+\cdots+\left(f_{z} *(x)-f_{z} *(y)\right)^{2}}
$$

Here, $f_{1}^{*}$ Is the $i t h$ normalized objective, the details of normalization are shown in $e_{x y}$. The teachers teaching $\Omega^{*}$ is made up of the non-dominated solution derived from the various algorithms.

The smaller the value of $E J_{R}$ the better the solutions of $\Omega^{l}$ and metric y indicates the ratio of the number of students to teachers' ratio in the set $\left\{x \in \Omega^{i} \mid x \varepsilon \Omega^{*}\right\}$ to $\Omega^{l}$.

The performance analysis of students derived from the hybrid system management algorithm is explained in Fig. 3. Further, it makes the majority of institutions to use this algorithm for online course administration. Many learning problems are formulated as a loss function reduction, which expresses the difference between the model's predictions

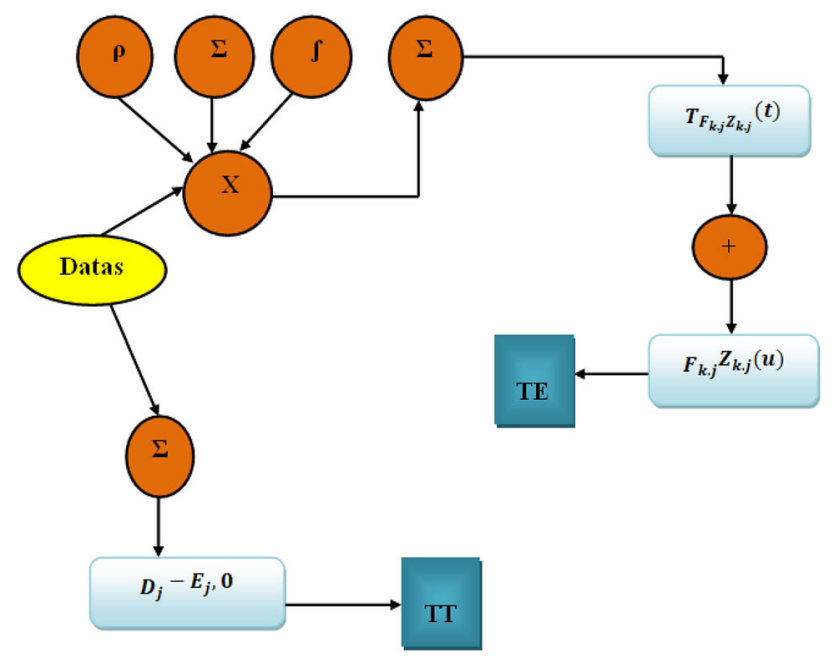

Fig. 4 TE and TT measurement being trained and the real instances of the problem. It has demonstrated that this highly active process has increased test scores in technical domains from 50 to $70 \%$ and reduced the drop-off rate by $22 \%$. Additional models can be validated by precise estimation techniques such as the remaining member method, which divides data into a training and test set and evaluates the test set training model's performance. Other educators can assess student understanding using mobile devices, which offer updates on student development in real-time and enable teachers to adapt and personalize their teaching. Equations (3) and (4) depicts the energy and delay used to improve students' performance and capacity, where TE refers to total power, and TT is total tardiness. This equation's primary goal is to assign jobs to teachers at the appropriate phases, identify the work sequence at each stage, and select an appropriate speed for each operation to minimize TT and maximize TE.

$g_{1}=T E=\sum_{l=j}^{n} \sum_{k=1}^{T_{F}} \int_{0}^{D_{\max }}\left(\sum_{j=1}^{e} F_{k . j} Z_{k . j}(u)+T_{F_{k . j} Z_{k . j}}(t)\right) d t$

$g_{2}=T T=\sum_{i=0}^{n}\left(D_{j}-E_{j}, 0\right)$

From Eq. (4) $Z_{k . j}(t)$ is a binary variableis mentioned. If $T_{F}$ works at the speed at time t, then $Z_{k . j}(t)$ is 1 ; otherwise $Z_{k . j}(t)$ is $0 . D_{\max }$ is the maximum completion time.If a teacher works at speed $k l$ at time t, then $F_{k . j} Z_{k . j}(u)$ is 1 ; otherwise $F_{k . j} Z_{k . j}(u)$ is $0 . D_{j}, E_{j}$ indicate the completion time and due date of the teacher's job. Metric $i$ and $n$ from Eq. (4) is the number of students and teachers included in the test phase.

Figure 4 shows the teacher problem and jobs' objective at the appropriate stages, defines the sequence of positions for each student and selects a reasonable speed to minimize TT and maximize the TE learning process. 


\subsection{Online supervision}

The study of computer algorithms that enhance experience automatically is machine learning (ML). Artificial intelligence is considered a component of predicting or decision making without explicit programming; machine learning algorithms construct a model based on sample data, referred to as training data. Machine teaching algorithms are used in various applications, including individual mail filtering and computer vision. It is difficult or impossible to develop traditional algorithms to perform the required tasks; therefore, multiple study algorithm is designed to make the training and teaching algorithms better.

Figure 5 shows a supervisory segmentation to enhance student learning and improve students' scores for better results and accuracy. Unmonitored learning (UL) is a type of algorithm that uses untagged data to learn patterns. The hope is that the machine must build a compact inner representation of its world through imitation. Half-supervised learning is a machine learning approach that combines small amounts of labelling data with numerous unlabeled data.

In machine learning, supervised learning is the job of creating a function using labeled training data. The training outcomes are already known; the system only has to learn how to get them appropriately. Standard approaches provide a classification system with extensive reference, encompassing the training data and the training results, by working as two vectors. The method uses inductive reasoning to generalize the training data. Unattended feature learning creates features from unlabeled input data, including dictionary learning, auto encoding, matrix factorization, and numerous cluster kinds.

Supervised learning is the task in machine learning to draw a function from labelled training data. The training results are previously known; the system learns how to obtain these results correctly. Standard procedures work as two vectors and produce a classification system with extensive reference, including the training data and the training outcome. The approach generalizes the training data, usually with inductive reasoning. Features are realized with unlabelled input data in unattended feature learning, with examples include dictionary learning, autoencoding, factorization of the matrix and various cluster types.

Semi-supervised education fell from unattended knowledge to supervised learning (no labelling of training data with only labelled training data). This is a specific case of weak monitoring and therefore needs to improve for better performance in learning.

One of the most popular neural network models is the self-organization map (SOM). It is part of the competition network category. The Self-Organizing Map is based on uncontrolled learning, which means no human intervention during education is needed. Consequently, they need little knowledge of the features of the input data, either few or abundant.

Supervised learning is thus an easier way of learning, while unsupervised learning is a complicated method, and therefore Supervised learning principal advantage is that it enables you to collect data from the previous experience or produce data from it. Therefore, making the learning process very effective and efficient is a new concept to the Eq. (5). In this, a teacher passes his knowledge to learners and a solution $Y_{\text {new }}$ is obtained as follows

$Y_{\text {new }}=Y+\alpha-\left(Y_{\text {teacher }}-U_{g} * Y_{\text {mean }}\right)$

As explored in Eq. (5) $Y_{\text {teacher }}$ teachers' knowledge is indicated. $U_{g}$ is a teaching factor and can be either 1 or 2 , and $Y_{\text {mean }}$ is the current mean value of all solutions, $\alpha$ denotes a random number following the uniform distribution on $[0,1]$. In the learning phase, learners increase their knowledge through interaction and are updated by the teacher. The updated knowledge of the students $Y_{\text {new }, i}$ is delivered by the following Eq. (6)

$Y_{\text {new }, i}=\left\{\begin{array}{c}Y_{i}+\alpha\left(Y_{i}-Y_{j}\right) \text { if } f\left(Y_{i}\right)<f\left(Y_{j}\right) \\ Y_{i}+\alpha\left(Y_{j}-Y_{i}\right) \text { otherwise }\end{array}\right.$

As described in Eq. (6) $Y_{j}, Y_{i}$ is a randomly selected and mainly selected learner for a hybrid learning management system. In the above phases, $Y_{\text {new }}$ or $Y_{\text {new }, i}$ are accepted if they give a better objective value than $Y$ or $Y_{i}$. Consequently, to improve the skills, learning speed, concentration, knowledge towards the field etc., is done using segmentation.

A high adhesive score for a segment indicates a sentence that appears "more than by chance," and further splitting the word collocation or semantic meaning of the words might break and in the following Eq. (7), the optimum segmentation is defined.

$\arg \max _{t 1 \ldots . t m} \sum_{i=1}^{m} D\left(t_{i}\right)$

As shown in Eq. (7) where $\underset{t 1 \ldots t m}{\max }$ is the maximum number of teachers, $D\left(t_{i}\right)$ is teachers' dependency towards the technology and principles, and metric $m$ denotes the maximum students. Dynamic programming with time complexity can derive the optimal segmentation.

As tweet segmentation's key is to extract meaningful phrases, longer segments are preferred for preserving more topically specific meanings. Therefore Eq. (8), which captures the factor: Length normalization, is derived as follows:

$D(s)=M(s) \cdot e^{Q(s)} \cdot \frac{2}{1+e^{T D(s)}}$

where $M(s)=1 i f|t|=1$ and. 


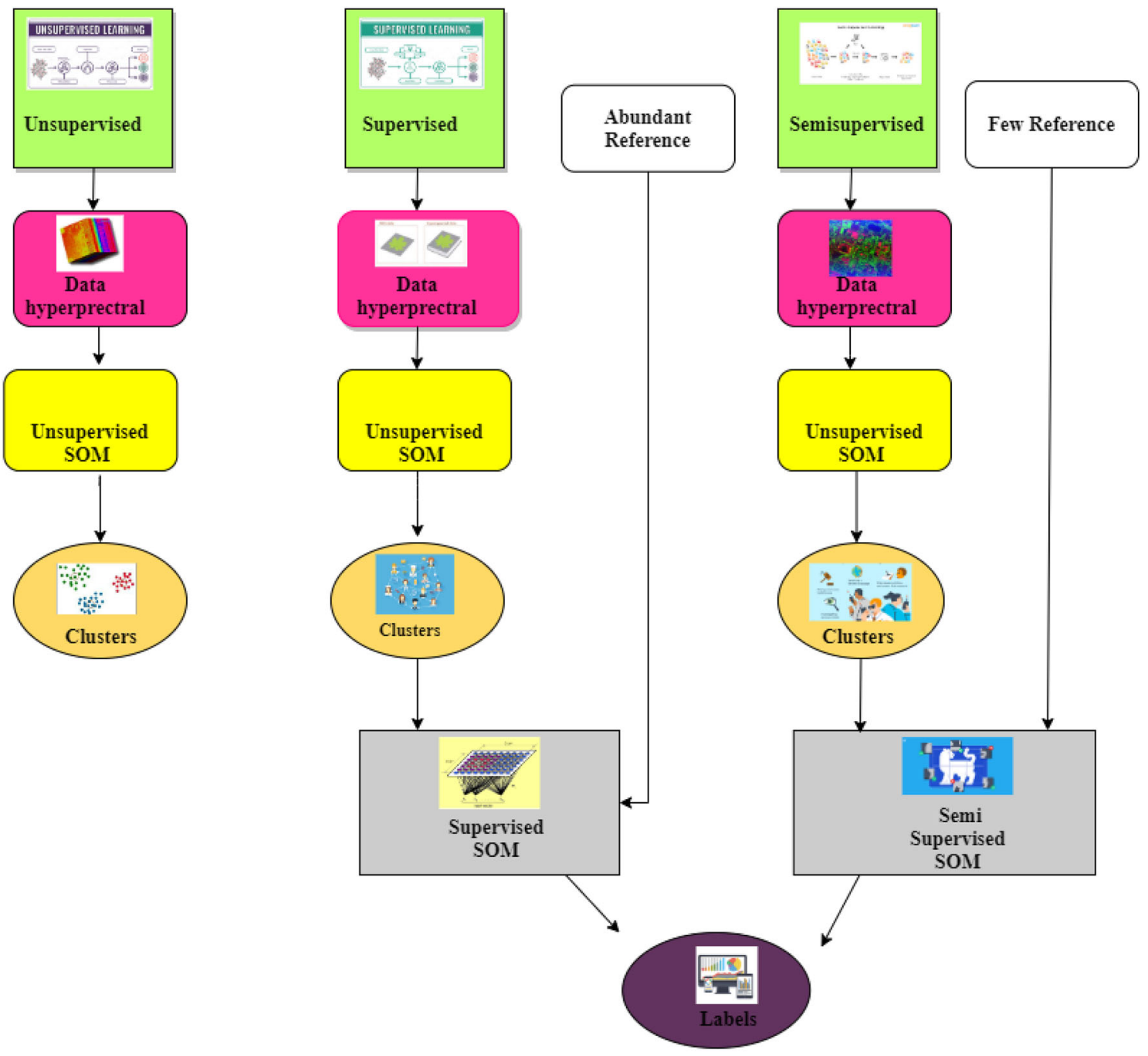

Fig. 5 Segmentation in Supervision

$M(s)=\frac{|t|-1}{t} \mathrm{if}|t|>1$,

which moderately alleviates the segments, and the metric $\mathrm{t}$ denotes the time component.

The second factor $e^{Q(s)}$ reveals optimization in learning, and the last component $\frac{2}{1+e^{T D(s)}}$ is to estimate the probability of a part in a useful phrase using Teachers dependency (TD) measure, and is defined in Eq. (9) as follows:

$$
T D(t)=\log \frac{Q_{r} s^{2}}{\frac{1}{|s|} \sum_{i=0}^{|s|-1} Q_{r}\left(w_{1} \ldots . w_{i}\right) Q_{r}\left(w_{i+1} \ldots \ldots w_{|s|}\right)}
$$

As defined in Eq. (9) $Q_{r} S$ or $Q_{r}\left(w_{1} \ldots \ldots w_{i}\right)$ is the approximated probability of a segment in Teachers dependency has been modified. If $\mathrm{t}$ contains a single word $T D(t)=2 \log Q_{r}$ $\left(w_{1}\right)$,. The estimation of $Q_{r} S$ is a critical challenge in our framework. In the following, two observations are considered, which are mainly the rationales $Q_{r} S$ can be estimated from global contexts, and $\frac{1}{|s|}$ from local contexts. Here, an approximate probability of $t$ being a good segmentation is defined by Eq. (10)

$Q_{r} \operatorname{seg} S=x(s, m) \cdot \frac{1}{m} \sum_{i}^{m} Q_{r_{r_{i}}}(s)$

where $x(s, m)=1 /\left(1+e^{-\alpha}\left(g_{x}^{r}-m / 2\right) \quad Q_{r_{r_{i}}}(s)=\right.$ $\left(1+\frac{\alpha^{\prime}}{g_{r, s}}\right)^{2}$

There are two parts to our approximation the first part from Eq. (10) depicts $x(s, m)$ the optimized knowledge and the second part $Q_{r_{r_{i}}}(s)$ for improved students and surveillance. Factor $1 /\left(1+e^{-\alpha}\left(g_{x}^{r}-m / 2\right)\right.$ is used to find good segmentation, and the metric $\alpha$ is the dependency factor based on 
Fig. 6 Monitoring by segmented supervision

Fig. 7 Performance analysis
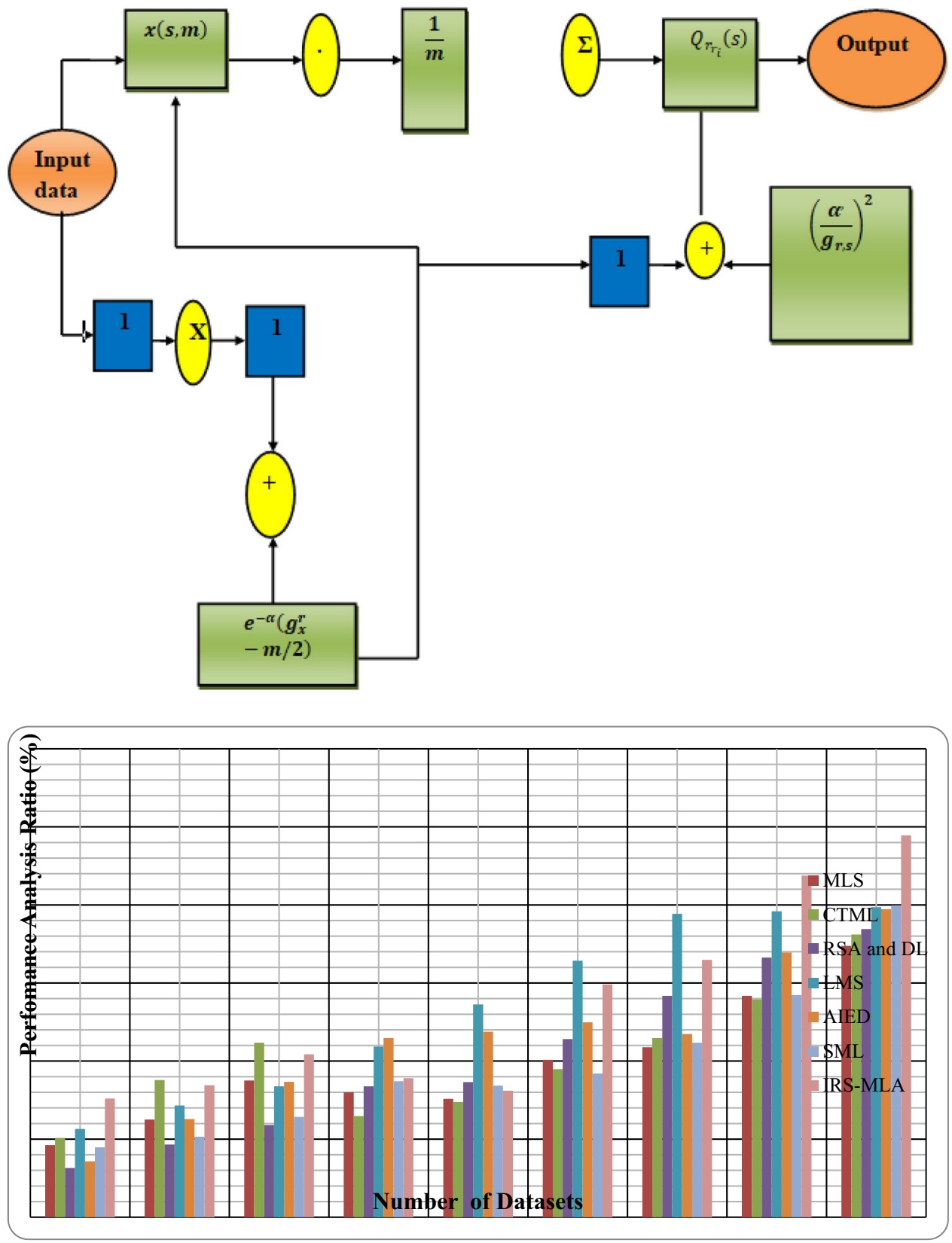

time. The average trust that a weak seg recognizes as an entity of segmentation is the correct quantity, and therefore, good segmentation is obtained. Figure 6 depicts segmentation and further improves learning skills enhanced algorithm is needed.

Furthermore, the enhanced monitoring by segmented control with an integrated remote control algorithm is inferred from Eq. (11)

$$
Z(s)=e^{R(s)} \cdot \rho_{s}
$$

With the weighting $Z(s)$, the top segments are chosen as enhanced learning and $e^{R(s)}$ is a rationalized factor. The probability of segment $\rho$ is calculated as followed in Eq. (12)

$$
\rho_{s}=\frac{\sum_{i}\left[e_{i, j}^{*}\right]}{|s| \cdot f_{s}} \cdot \frac{1}{1+e^{-5 \frac{f_{x}-f^{*} x^{2}}{f}}}
$$


Table 1 Comparative performance analysis

\begin{tabular}{llllllll}
\hline Number of datasets & MLS & CTML & RSA and DL & LMS & AIED & SML & IRS-MLA \\
\hline 10 & 18.45 & 20.3 & 12.6 & 22.6 & 14.3 & 17.9 & 30.4 \\
20 & 25 & 35.12 & 18.6 & 28.6 & 25.14 & 20.6 & 33.8 \\
30 & 35 & 44.67 & 23.6 & 33.5 & 34.67 & 25.7 & 41.7 \\
40 & 32 & 25.9 & 33.5 & 43.7 & 45.9 & 34.8 & 35.6 \\
50 & 30.3 & 29.5 & 34.6 & 54.5 & 47.5 & 33.7 & 32.4 \\
60 & 40.3 & 37.9 & 45.6 & 65.7 & 49.9 & 36.8 & 59.6 \\
70 & 43.5 & 45.9 & 56.7 & 77.7 & 46.9 & 44.7 & 65.9 \\
80 & 56.7 & 55.8 & 66.5 & 78.35 & 67.8 & 56.9 & 87.5 \\
90 & 69.5 & 72.4 & 73.8 & 79.4 & 78.9 & 79.9 & 97.8 \\
\hline
\end{tabular}

Table 2 Comparative analysis of efficiency

\begin{tabular}{llllllll}
\hline Number of datasets & MLS & CTML & RSA and DL & LMS & AIED & SML & IRS-MLA \\
\hline 10 & 15.34 & 18.45 & 14.45 & 30.44 & 14.4 & 40.33 & 59.29 \\
20 & 23.4 & 38.56 & 30 & 45.8 & 28.7 & 46.9 & 70.34 \\
30 & 35.56 & 60.56 & 42.56 & 47.9 & 32.56 & 50.6 & 81.46 \\
40 & 33.89 & 55.7 & 45.56 & 48.4 & 35.44 & 62.6 & 70.67 \\
50 & 31.54 & 73.7 & 49.89 & 55.6 & 23.66 & 65.65 & 82.62 \\
60 & 43.3 & 74.56 & 51.98 & 63.56 & 34.67 & 68.9 & 92.45 \\
70 & 44.45 & 86.9 & 60.45 & 65.33 & 38 & 84.3 & 96.34 \\
80 & 54.7 & 89 & 67.67 & 74.54 & 45.43 & 86.4 & 96.76 \\
90 & 66.56 & 90.5 & 70.56 & 76.56 & 60.11 & 90.4 & 99.32 \\
\hline
\end{tabular}

As explored in Eq. (12), two factors are considered; the first factor estimates the probability of the student's performance, and the second factor gives the enhanced result in learning due to supervision and these factors are used to improves the score. Here, the elements are defined in two consecutive iterations as followed in Eqs. (13) and (14)

$Q_{t}^{s}(s)=\left(1+\frac{\alpha}{t_{s}^{i}+}\right)^{\frac{t_{x}}{t_{x+i}^{i}}}$

Suppose at iteration $I$ segmented outputs a set of segments, where $t_{x}^{i}$ is the number of times $x$ is a segment at iteration $i$. Then, $\frac{\alpha}{t_{+}^{i}+}$ relatively records the segmentation confidence of HybridSegmentation about $s$ at iteration $i$.Similar to Eq. (13), have the following iterative updating function:

$Q_{t}^{s+1}=(1-\gamma) Q_{t_{i}}+\gamma Q_{t}^{s}(\mathrm{~s})$

In the 0 th iteration, $Q_{t}^{s+1}$ can be estimated based on the learners' performance, where $\gamma$ is the coupling factor in Eq. (14) is crucial for the convergence of segments. A good should ensure that the top confident segments from the previous iteration are detected more times in the next iteration. Accordingly, learning the parameter is converted to an optimization problem with new technology and, the efficiency is increased instead is not a significant concern for a fixed number of values.

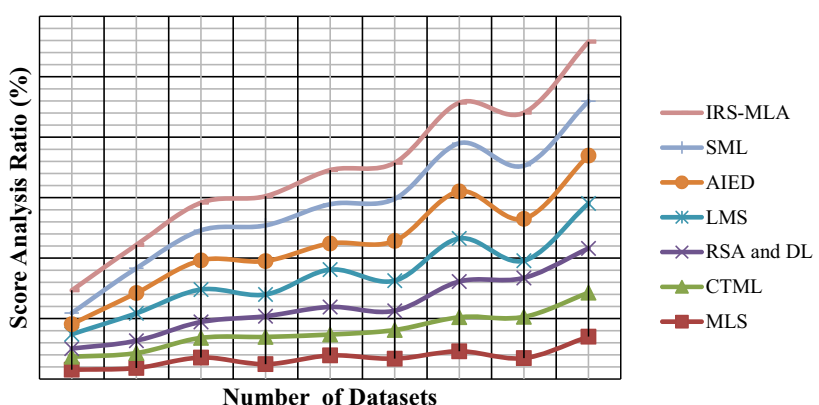

Fig. 8 Improved score analysis

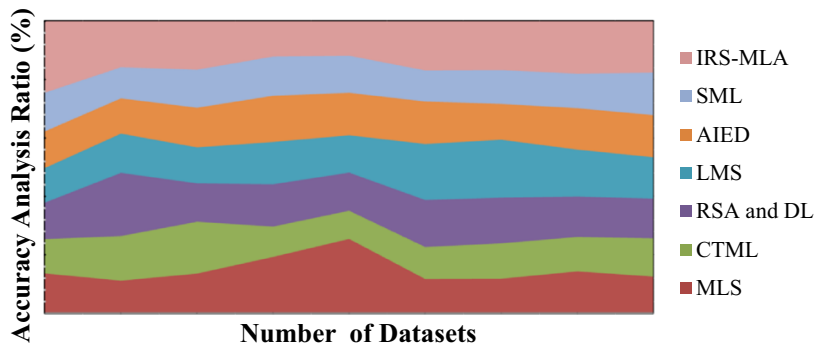

Fig. 9 Accuracy analysis

Machine-learning in some areas has been transformative; consequently, machine-learning programmes often fail to produce expected results. There are many causes for this: the absence of (suitable) data, the lack of access to the data, 
Fig. 10 Efficiency analysis

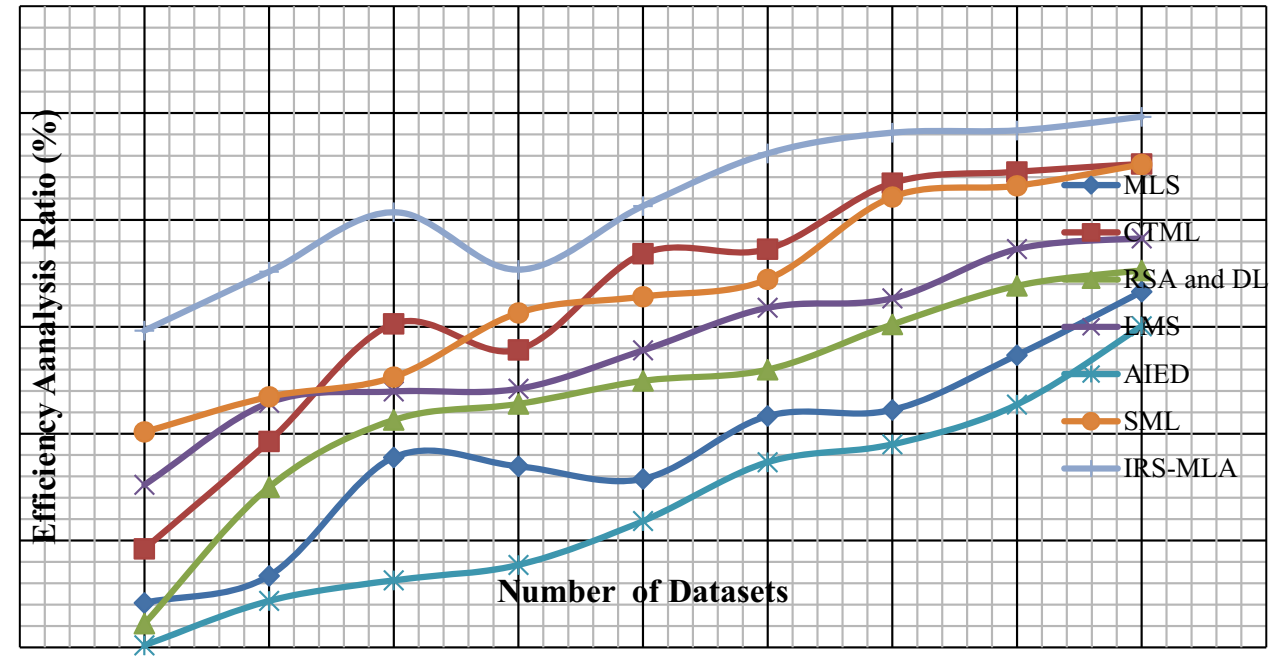

Fig. 11 Ratio of success analysis

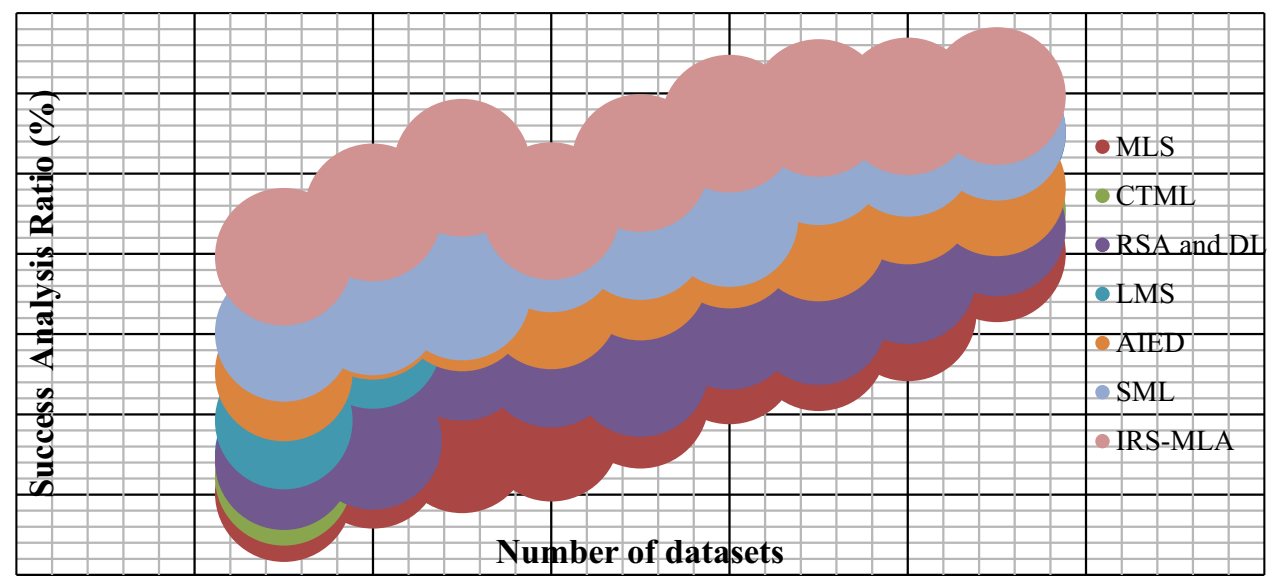

data differentiation, privacy issues, mistaken tasks and algorithms, the absence of resources and evaluation issues.

Therefore to reduce this problem and enhance the learning process, the technique IRS-MLE algorithms is proposed. The IRS-MLA is used to update the evidence relating to systematic examination and increased review burden in teaching growth. Although the training sets have improved, they have not yet sufficiently reduced the workload without limiting the sensitivity required for research findings themselves.

\section{Results and discussion}

The data used are generated by the random generation of data in a fixed range of values, showing the number of observations made with particular students' particular attention; for example, the number of hands raised and the number of eyebrows increased. For this study, the large dataset from Kaggle [27] is used, and the dataset is further split into multiple experimental groups of 9 different datasets with multiple sets of components.
Figure 7 shows mainly the data generated to enhance student performance assessment. When the data were grouped and identified, the algorithm would be used to classify them.

The continuous performance improvement aspect of learning is embedded within the integrated remote supervision algorithm and allows all levels of instruction and assessment to support each other.

Table 1 predicts the validation outcome at greater than $50 \%$ for any of the datasets at any cross-validation granularity. Therefore, inverting the IRS-MLA algorithm's outcome can predict the unseen data at the increased performance. The performance comparative study showed that MLS,CTML,RSA and DL, LMS,AIED,SML, and IRS-MLA have a performance ratio of $69.5 \%, 72.4 \%, 73.8 \%, 79.4 \%$, $78.9 \%, 79.9 \%$ and $97.8 \%$, respectively. This is a key result whereby the IRS algorithm's importance is highlighted, and performance is improved by $97.8 \%$ Table 2 .

The information was classified using the IRS-MLA algorithm and the HLMS algorithm. The formal and informal evaluation forms, including groups, student portfolios, and class participation, assess student learning. 
The final student classification and the associated boundary of decision are shown in Fig. 8. The score out of 600 was evaluated and observed the highest score in percentage with a $97 \%$ score for the dataset 9 . Students cannot observe these results in the initial course or test set and automatically classified carefully or carelessly, based on clustering and classification. Moreover, the score increases quickly with this classification of students.

The emphasis on model performance is elegantly taught on data excluded from the training process. As this paper shows, constructing a model that reflects the data it has been trained with is insignificant. Figure 9 illustrate the predictive and defined validation data using ground reality results to divide the relevant retrieval documents into fractions. The accuracy metric is more generally used to evaluate the learning abilities of students.

A fourth problem is the level of control objectives in the desired output values. If the desired output values are often wrong, the learning algorithm should not locate a function that corresponds precisely to the workouts. To try to fit the data too carefully leads to better performance. Figure 10 improves the desired output values with the training data subset and labelling of the remaining data.

The proposed method for improving student performance is illustrated in Table 1. It means optimal output using the lowest input to generate the highest performance. The comparative research outcome suggests that the efficiency rate is $66.56 \%, 90.5 \%, 70.56 \%, 76.56 \%, 60.11 \%, 90.4 \%$ for MLS, CTML, RSA and DL, LMS, AIED, SML, and IRS-MLA. The research still gave a better approach, with an improved efficiency rate of $99.3 \%$.

Results demonstrate that the educational systems can be evaluated on the successful transmission of knowledge and skills. To improve student learning is an objective in educational reform, HLMS aims at increasing the number of individualized interactions between students and teachers. HLMS is said to it has been a reform in numerous districts with a long theoretical appeal, being the best success in the educational field. The simulation results show that integrated remote supervision achieves a performance of $97.8 \%, 97 \%$ score, an accuracy of $99.32 \%$, an efficiency of $96 \%$, and a success ratio of $98 \%$.

\section{Conclusion}

An online teaching experience involves a range of computerized teaching procedures, including computer resources and software. Besides, a learning process is enhanced effectively using integrated remote supervision with machine learning algorithms (IRS-MLA). Therefore, the people learn to advance for collaborative projects and concentrate on assess- ment and feedback. Furthermore, students performance is increased.

Further, introducing an electronic tracking system between teachers and their supervisors can interact more effectively. (E-Supervision). E-Supervision refers to the use of videoconferencing technologies in real-time management. Since the e-place supervisors are physically different from their supervisors, the e-supervisor may perform various professional tasks with video conference technologies. Methods under supervision will enable the supervisor to improve professionally and continue the supervisor's growth and education. As a result, using analytical data and reporting, a hybrid learning management system (HLMS) addressed the training and learning gaps between teachers and students. The simulation results demonstrated that the proposed integrated remote supervision improved the proposed learning management system by $97.8 \%, 97$ percent score, 99.32 percent accuracy, 96 percent efficiency, and 98 percent success. This, therefore, provided an environment of educational collaboration among supervisors and teachers, with the addition of skills, experiences, attitudes, and teaching policies.

Acknowledgments This work is supported by the Teaching Reform Project of Guangxi Higher Education (Grant No. 2020JGZ117). This work is supported by the Planning Project of Guangxi Education Sciences: Research on the Long-term Mechanism and Implementation Path of Guangxi Minority Culture Inheritance Education through Cooperation between University and Secondary Vocational School (Grant No. 2019ZJY111).

\section{References}

1. Hartman, J., Morris, K.: Developing an effective interactive online educational leadership supervision course. Int. J. Teach. Learn. Higher Edu. 31(3), 524-535 (2019)

2. Arooj, A., Farooq, M. S., Akram, A., Iqbal, R., Sharma, A., \& Dhiman, G.: Big Data Processing and analysis in internet of vehicles: Architecture, taxonomy, and open research challenges. Archives of Computational Methods in Engineering, (2021) 1-37

3. Manogaran, G., Alazab, M., Saravanan, V., Rawal, B.S., Shakeel, P.M., Sundarasekar, R., Montenegro-Marin, C.E.: Machine learning assisted information management scheme in service concentrated IoT. IEEE Trans. Ind. Informatics 17(4), 2871-2879 (2020)

4. Hakak, S., Alazab, M., Khan, S., Gadekallu, T.R., Maddikunta, P.K.R., Khan, W.Z.: An ensemble machine learning approach through effective feature extraction to classify fake news. Future Gener. Comput. Syst. 117, 47-58 (2021)

5. Kayapinar, U., Spathopoulou, F., Safieddine, F., Nakhoul, I., Kadry, S.: Tablet use in teaching: A study on developing an attitude scale for academics. Eurasian J. Edu. Res. 18(78), 219-234 (2018)

6. Liao, H., Zhou, Z., Zhao, X., Zhang, L., Mumtaz, S., Jolfaei, A., Bashir, A.K.: Learning-based context-aware resource allocation for edge-computing-empowered industrial IoT. IEEE Internet Things J. 7(5), 4260-4277 (2019)

7. Kumar, D., Sharma, A., Kumar, R., \& Sharma, N.: Restoration of the network for next-generation (5G) optical communication 
network. In 2019 International Conference on Signal Processing and Communication (ICSC) (pp. 64-68). (2019) IEEE

8. Fan, M., \& Sharma, A.: Design and implementation of construction cost prediction model based on SVM and LSSVM in industries 4.0. International Journal of Intelligent Computing and Cybernetics (2021)

9. Elhoseny, M.: Multi-object detection and tracking (MODT) machine learning model for real-time video surveillance systems. Circuits, Syst., Signal Process. 39(2), 611-630 (2020)

10. Kanisha, B., Lokesh, S., Kumar, P.M., Parthasarathy, P., Babu, G.C.: Speech recognition with improved support vector machine using dual classifiers and cross fitness validation. Pers. Ubiquitous Comput. 22(5), 1083-1091 (2018)

11. Shakeel, P. M., Baskar, S., Fouad, H., Manogaran, G., Saravanan, V., \& Xin, Q.: Creating collision-free communication in IoT with $6 \mathrm{G}$ using multiple machine access learning collision avoidance protocol. Mobile Networks and Applications, (2020) 1-12

12. Nieto, Y., García-Díaz, V., Montenegro, C., Crespo, R.G.: Supporting academic decision making at higher educational institutions using machine learning-based algorithms. Soft Comput. 23(12), 4145-4153 (2019)

13. Khamparia, A., Singh, S.K., Luhach, A.K., Gao, X.Z.: Classification and analysis of users review using different classification techniques in an intelligent e-learning system. Int. J. Intell. Inf. Database Syst. 13(2-4), 139-149 (2020)

14. Balaanand, M., Karthikeyan, N., Karthik, S., Varatharajan, R., Manogaran, G., Sivaparthipan, C.B.: An enhanced graph-based semi-supervised learning algorithm to detect fake users on Twitter. J. Supercomput. 75(9), 6085-6105 (2019)

15. Ramesh, S., Yaashuwanth, C., Muthukrishnan, B.A.: Machine learning approach for secure communication in wireless video sensor networks against denial-of-service attacks. Int. J. Commun. Syst. 33(12), e4073 (2020)

16. Bhalaik, S., Sharma, A., Kumar, R., Sharma, N.: Performance modeling and analysis of WDM optical networks under wavelength continuity constraint using MILP. Recent Adv. Electr. Electron. Eng. (Former. Recent Pat. Electr. Electron. Eng.) 13(2), 203-211 (2020)

17. Sun, H., Fan, M., \& Sharma, A.: Design and implementation of construction prediction and management platform based on building information modelling and three-dimensional simulation technology in industry 4.0. IET Collaborative Intelligent Manufacturing (2021)

18. Murphy, E., Rodríguez-Manzanares, M.A., Barbour, M.: Asynchronous and synchronous online teaching: Perspectives of Canadian high school distance education teachers. British J. Edu. Technol. 42(4), 583-591 (2011)
19. Chen, R., \& Sharma, A.: Construction of complex environmental art design system based on 3D virtual simulation technology. International Journal of System Assurance Engineering and Management, (2021) 1-8

20. Pang, H., Zheng, Z., Zhen, T., Sharma, A.: Smart farming: An approach for disease detection implementing IoT and image processing. Int. J. Agric. Environ. Inf. Syst. (IJAEIS) 12(1), 55-67 (2021)

21. Choudrie, J., Banerjee, S., Kotecha, K., Walambe, R., Karande, H., Ameta, J.: Machine learning techniques and older adults processing of online information and misinformation: A covid 19 study. Comput. Human Behav. 119, 106716 (2021)

22. Zhai, X., Yin, Y., Pellegrino, J.W., Haudek, K.C., Shi, L.: Applying machine learning in science assessment: a systematic review. Stud. Sci. Edu. 56(1), 111-151 (2020)

23. Yongqing, H. Evaluation of English online teaching based on remote supervision algorithms and deep learning. Journal of Intelligent \& Fuzzy Systems, (Preprint), 1-12

24. Villegas-Ch, W., Román-Cañizares, M., Palacios-Pacheco, X.: Improvement of an online education model with the integration of machine learning and data analysis in an LMS. Appl. Sci. 10(15), 5371 (2020)

25. Zawacki-Richter, O., Marín, V.I., Bond, M., Gouverneur, F.: Systematic review of research on artificial intelligence applications in higher education-where are the educators? Int. J. Edu. Technol. Higher Edu. 16(1), 1-27 (2019)

26. Wu, J.Y., Hsiao, Y.C., Nian, M.W.: Using supervised machine learning on large-scale online forums to classify course-related Facebook messages in predicting learning achievement within the personal learning environment. Interact. Learn. Environ. 28(1), 65-80 (2020)

27. https://www.kaggle.com/c/student-academic-performanc

Publisher's Note Springer Nature remains neutral with regard to jurisdictional claims in published maps and institutional affiliations. 